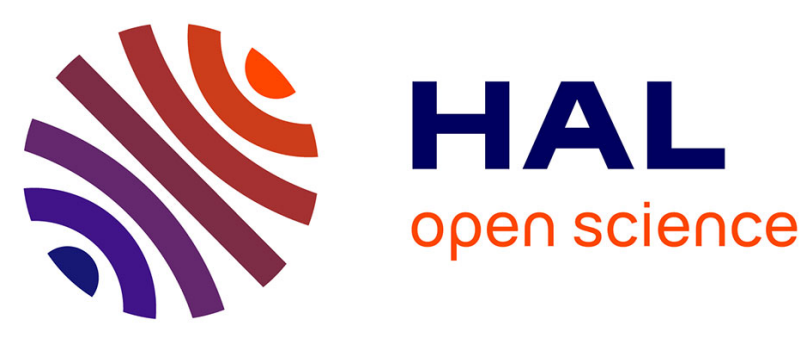

\title{
D'un renoncement au père
}

\author{
Fethi Benslama
}

\section{To cite this version:}

Fethi Benslama. D'un renoncement au père . Topique - Revue freudienne, 2003, Les spiritualités, 4 (85), pp.139-148. 10.3917/top.085.0139 . hal-01502515

\section{HAL Id: hal-01502515 https://hal.science/hal-01502515}

Submitted on 5 Apr 2017

HAL is a multi-disciplinary open access archive for the deposit and dissemination of scientific research documents, whether they are published or not. The documents may come from teaching and research institutions in France or abroad, or from public or private research centers.
L'archive ouverte pluridisciplinaire HAL, est destinée au dépôt et à la diffusion de documents scientifiques de niveau recherche, publiés ou non, émanant des établissements d'enseignement et de recherche français ou étrangers, des laboratoires publics ou privés. 


\section{D'UN RENONCEMENT AU PÈRE}

Fethi Ben Slama

L’Esprit du temps | « Topique »

2003/4 nº 85 | pages 139 à 148

ISSN 0040-9375

ISBN 2847950184

Article disponible en ligne à l'adresse :

http://www.cairn.info/revue-topique-2003-4-page-139.htm

\section{Pour citer cet article :}

Fethi Ben Slama, « D'un renoncement au père », Topique 2003/4 ( $\left.\mathrm{n}^{\circ} 85\right)$, p. 139-148.

DOI 10.3917/top.085.0139

Distribution électronique Cairn.info pour L'Esprit du temps.

(C) L'Esprit du temps. Tous droits réservés pour tous pays.

La reproduction ou représentation de cet article, notamment par photocopie, n'est autorisée que dans les limites des conditions générales d'utilisation du site ou, le cas échéant, des conditions générales de la licence souscrite par votre établissement. Toute autre reproduction ou représentation, en tout ou partie, sous quelque forme et de quelque manière que ce soit, est interdite sauf accord préalable et écrit de l'éditeur, en dehors des cas prévus par la législation en vigueur en France. Il est précisé que son stockage dans une base de données est également interdit. 


\title{
D’un renoncement au père
}

\author{
Fethi Benslama
}

L'islam aurait-il tenté de produire à l'intérieur de son édifice spirituel une réduction de la nostalgie du père, un renoncement à sa figure pour constituer la foi en Dieu? Je pose cette question au terme d'une recherche qui m'a conduit à une exploration des textes et des constructions symboliques de la religion islamique, au regard des hypothèses de la psychanalyse ${ }^{1}$.

À première vue, cette formulation semble contredire la thèse constante de Freud, de penser la Sehnsucht du père ${ }^{2}$ à la racine de la création des dieux et des formations religieuses. Mais la recherche psychanalytique sur la culture doitelle se contenter d'appliquer avec une fidélité iconique, la lecture freudienne, lorsque les faits viennent compliquer l'extension compréhensive des ressorts de la psychologie individuelle vers la vie collective? D'autant que, dans ses développements sur le monothéisme, Freud avait laissé de côté le cas de l'islam qu'il mentionne très rapidement sous le chapitre «des difficultés » dans L'homme Moïse et la religion monothéiste ${ }^{3}$, pour proposer une interprétation de son système spirituel autour de la question du père, qui s'avère très problématique à un examen minutieux. ${ }^{4}$

On se souvient que dans Un souvenir d'enfance de Léonard de Vinci, Freud évoque la belle phrase de Léonard: «Qui s'appuie dans la controverse sur l'autorité ne travaille pas avec l'esprit mais avec la mémoire $»^{5}$, pour la

1. Fethi Benslama, La psychanalyse à l'épreuve de l'islam, Paris, Aubier, 2002.

2. Traduite par «désirance pour le père », S. Freud, Totem et Tabou (1912-13), O C. XI, p. 367.

3. S. Freud, L'homme Moïse et la religion monothéiste (1939), trad. C. Heim, Paris, Gallimard, 1986, p. 186.

4. Cf. «La discussion de la formulation de Freud», in F. Benslama, op. cit., pp. 115-120.

5. S. Freud, Un Souvenir d'enfance de Léonard de Vinci, (1910), trad. M. Bonaparte, Paris, Gallimard, 1977,p. 123. 
commenter assez longuement et proposer l'hypothèse selon laquelle l'une de sources de la liberté dont témoigne l'œuvre de Léonard, au regard de son époque, réside dans le fait qu'il ait « appris à renoncer au père » dès l'enfance, et n'eut plus besoin tout au long de sa vie de s'appuyer sur la transfiguration du père en Dieu, pour mener ses recherches audacieuses jusqu'à s'éloigner « de la conception chrétienne de l'univers ». Freud ne transforme pas pour autant Léonard en un personnage sans foi, puisqu'il souligne combien il «ne manque pas d'exprimer son admiration pour le Créateur, cause première de tous ces mystères merveilleux $(. ..){ }^{6}{ }^{6} \mathrm{Il}$ indique cependant, que le renoncement au père a ouvert un espace de «jeux » et «d'écarts » pour son imagination (les mots sont de Freud) qui amène l'esprit à faire de la nature le lieu de sa propre recherche, et termine son étude en attribuant à ce trait de caractère de Léonard, la mention dans ses manuscrits milanais, d'une adoption de la religion musulmane.

Il ne s'agit pas de prétendre que l'édifice spirituel de l'islam se passe de la question du père, ni de lui attribuer une capacité de liberté dont l'histoire réelle nous montre les limites, à l'instar de toutes les religions et de toutes les constructions dogmatiques. L'hypothèse que je souhaite instruire, consiste plutôt à exposer une conjoncture à travers laquelle, il apparaît que le fondateur de l'islam ( $\mathrm{VI}^{\mathrm{e}}$ siècle), ayant en vue le traitement que le judaïsme et particulièrement le christianisme réservèrent au rapport entre Dieu et le père, tenta un dépassement qui le conduisit à produire un impératif intenable, en un sens comparable à celui de l'amour chrétien du prochain, un commandement dont la sublimité le rendait insoutenable pour la psyché humaine. En somme, la séparation radicale entre le père et Dieu aurait le caractère «antipsychologique» qui revient à demander à l'homme de se tenir à l'impossible. Mais ne serait-ce pas sur cette limite qu'un ordre symbolique recèle la possibilité d'une liberté spirituelle susceptible d'engendrer des moments et des œuvres de sublimation dans la civilisation? Inversement, on peut se demander si l'assignation à l'impossible, sous certaines conditions historiques, notamment quand les forces herméneutiques manquent, n'entraîne pas vers un tourbillon de désespoir?

$$
-\mathrm{II}-
$$

La figure du père n'entre pas dans les constructions dogmatiques de l'islam. Dès l'origine, le Coran prend un soin particulier à éloigner la référence à Dieu de la représentation de la paternité, même à titre métaphorique ou allusif. Les proclamations de l'unicité de Dieu bannissent radicalement toute notion de génération ou d'engendrement divin. Dans la sourate dite du Culte pur, la nature divine est proclamée en ces termes abrupts :

«Dis : Lui Dieu l'Un. Dieu de la plénitude. N'engendre pas. N'est pas engendré. Nul n'est égal à Lui.» ${ }^{7}$.

\footnotetext{
6. Op. cit., p. 127.

7. Coran, CXII.
} 
Les commentateurs présentent ce passage comme une réfutation du DieuPère du christianisme. Le mot «plénitude» qui fut anciennement le titre de cette sourate vise à excepter Dieu de l'ordre du sexe et de la génération. Il s'agit de la traduction du terme «Çamad» qui désigne ce qui est plein et complet, soit «l'impénétrable». Cette dernière signification a prévalu à travers de nombreuses traductions ${ }^{8}$. La complétude divine s'oppose en effet, formellement, à la nature ouverte de l'homme, ouverte parce que sexuée, sexuée parce que fendue, puisque le sexe est le «Farj» qui est trou, interstice, défaut ${ }^{9}$.

Certaines versions du mythe de la création font du sexe l'élément inaugural du façonnage de l'homme: «Ce que Dieu a créé de premier en l'homme écrit Qortobî, ce fut son sexe (Farj), il dit: "ceci est mon dépôt, je vous le confie", car le sexe est un dépôt (Amânatun).» ${ }^{10}$. Le dépôt revêt ici le sens d'un objet inestimable qui marque l'insigne dignité de l'homme. Le terme arabe est de la même racine que l'Amen hébreu ; il désigne un « oui », un « ainsi soit-il » premier et corrélatif de la réception du manque originaire dans la conformation humaine. Mais cette aménité constitue en même temps la source du problème éthique central de l'homme, que Le Coran indique dans ces termes: "Nous avons proposé le dépôt (Amanata) aux cieux, à la terre et aux montagnes, ils ont refusé de s'en charger et ils s'en sont effrayés, sauf l'homme qui a accepté, car il est injuste et ignorant. ${ }^{11}$ Ainsi, le «oui» originaire au sexuel recèle une présomption sans égal.

Il existe d'autres versions de ce mythe qui représentent le corps primitif de l'homme, comme un corps troué, livré à travers ses orifices à la circulation démoniaque, en tant qu'être de feu : «Lorsque Dieu créa l'homme d'argile, en attendant qu'il lui insuffle une âme, Satan se moquait de lui en jouant à pénétrer par sa bouche, à sortir par son anus et l'inverse. Il empruntait de la même manière d'autres orifices: oreille, nez, etc.» ${ }^{12}$ À l'évidence, ce fragment met en scène une ironie pulsionnelle consubstantielle à la topique du trou comme sexuel originaire, dont il appartiendra à l'âme ou à la psyché de tenter de la circonvenir, en la couvrant à travers ses trois fonctions fondamentales: perception, imagination et intellection par la raison. Tous les traités sur la psyché, tel que chez Avicenne ${ }^{13}$, se meuvent à l'intérieur de ce paradigme quelles que soient les sophistications philosophiques qu'ils lui apportèrent au fil du temps. Le sexe apparaît bien comme le point aveugle (l'une des métaphore du sexe en arabe) de la rencontre avec la psyché.

\footnotetext{
8. Par exemple dans la traduction de Denise Masson, Coran, Paris, Gallimard, 1967.

9. Fethi Benslama, «Le sexe absolu », in Cahiers Intersignes. $\mathrm{N}^{\circ} 2$, Paris, 1991, pp. 105124.

10. Al-Qortubî, Al-jâma ' li 'ahkâm al-qur’ân, Le Caire, Éditions Al-Kutub Al-masryya, 1954, t XVI, p. 254.

11. Coran, VXXI, 33.

12. Ibn Ayyâs Al-Hanafî, Badâ' $i^{\prime}$ az-zuhûr fì wqâ' $i^{`}$ 'ad-duhûr, Tunis, éditions du Manâr, s. date, pp. 38-39.

13. Ibn Sîna (Avicenne), An-nafs (De anima), établi par I. Madkour, Le Caire, 1975.
} 
La séparation est donc radicale : d'un côté Dieu est hors sexe, hors génération; de l'autre côté, l'humanité façonnée autour du trou est au bord d'un abîme qui est la marque de la transcendance en elle. Elle manie l'imagination et la raison pour en réduire les périls et instaurer la jouissance sexuelle légale (Nikâh). Toute la spiritualité de l'islam va se tenir au plus près de cette séparation. Elle pose que là où il y a Dieu, il n'y a ni paternité, ni maternité, ni engendrement, ni rapport sexuel. Aucune métaphore ne peut franchir cet impossible.

Dans sa traduction du Coran, Jacques Berque a rapproché le passage du Culte pur cité plus haut, de l'une des premières définitions du dieu Un, dans le Poème de Parménide. La traduction de Marcel Conche du fragment 8 montre en effet, une proximité troublante avec le verset en question:

"[...] qu'étant inengendré, il est aussi impérissable, entier, unique, inébranlable et sans terme.

Ni il était une fois, ni il sera, puisqu'il est maintenant, tout entier ensemble, un continu. ${ }^{14}$

Le commentaire de Marcel Conche indique en quoi la complétude de ce dieu $U n$ «inengendré » établit l'idée d'un dieu radicalement différent de tout étant, un dieu qui est «être » auquel on ne peut ni retrancher ni ajouter. Saturé, il est impossible.

Quelque soit le résultat des recherches sur les sources grecques du Coran, qui permettront peut-être un jour de mieux préciser la nature exacte des transmissions et des traductions, il n'en demeure pas moins que la dernière religion monothéiste, née au $\mathrm{VI}^{\mathrm{e}}$ siècle, se présente d'emblée comme une objection à la théologie de la paternité divine, en installant un désert généalogique entre l'homme et Dieu. Les conséquences en sont multiples et à tous les nivaux. Sur le plan du développement historique d'abord, on se rappelle la thèse de Hegel qui attribue la rapidité avec laquelle l'islam devient « un empire universel», au degré élevé de «l'abstraction de son principe » et à «la plus haute intuition de l'Un» dans sa conscience ${ }^{15}$. Sur le plan philosophique, Christian Jambet a montré comment ce contenu de la foi amènera l'éclosion d'une ontologie qui établit une équation entre Dieu et être, entre l'Un et l'identité du réel ${ }^{16}$. Quant à la question du sujet, notre recherche commence à peine à entrevoir certaines implications de la foi en un Dieu qui est être et réel.

Dans des circonstances sur lesquelles je ne peux m'attarder, coupant court à la possibilité de la paternité adoptive qui existait chez les Arabes avant l'islam, Le Coran exclut aussi le fondateur de l'islam du statut de père: «Muhammad n'est le père d'aucun homme parmi vous $»{ }^{17}$. Si la médiation prophétique ne

14. Parménide, Le Poème : Fragments, trad. M. Conche, Paris, P.U.F., 1996, p. 127.

15. G.W.F. Hegel, La raison dans 1'histoire, 10/18, 1965, p. 293.

16. Christian Jambet, L'acte d'être, Paris, Fayard, 2003.

17. Coran, XXXIII, 40. 
revêt pas les attributs paternels, c'est pour autant que le premier des musulmans est placé d'emblée en position de fils et d'orphelin, puisque l'une des toutes premières apostrophes que Dieu a adressée au prophète, est celle par laquelle il l'appelle: «l'orphelin ». La relation de Dieu à l'homme ne passera pas donc par la médiation d'un prophète paternel. La paternité ne sera jamais comme dans le judaïsme au centre de l'alliance avec Yahvé, en tant que Dieu des pères.

De façon générale, il n'existe pas en islam de sacralisation du père, ni lors de la fondation de la nouvelle religion, ni dans l'histoire exégétique de sa transmission. Bien plus, le père est l'objet d'une distanciation, d'une critique insistante dont témoigne le texte coranique même. D'abord, on ne rencontre que quelques rares occurrences (sept fois) où le Coran évoque favorablement ce qu'il appelle « les pères premiers » (al-abâ' al-'awwalîn). Plusieurs commentateurs ont noté judicieusement que Le Coran et la parole du prophète (hadîth) n'usent pas du terme «père» au singulier, mais toujours au pluriel, comme s'il n'y avait pas $L e$ père comme principe ou comme essence. Il s'agit principalement d'Abraham, secondairement d'Ismaël, d'Issac et de Jacob dont l'évocation comme «pères » est mise dans la bouche d'un personnage biblique, plutôt qu'à travers une nomination directe. Dans la très grande majorité des cas, le pluriel «pères » renvoie à des faits, des figures ou des jugements négatifs. Les pères sont dans l'erreur, dans la déraison, ils succombent à la tentation, ils sont idolâtres et oublieux ; ils sont interpellés, dénoncés, appelés à croire au Dieu unique et quelques fois pardonnés pour leurs fautes.

Du reste, Abraham qui est la figure centrale de la paternité pour l'islam est présenté comme l'exemple même de la fondation du monothéisme à partir de la désobéissance au père, puisque Abraham refusera le culte polythéiste de son père Terah et le quittera pour accomplir une migration spirituelle. Celle-ci revêt le sens d'une libération de la loi du père, de son clan et de sa coutume; de sorte que l'esprit du monothéisme pour l'islam ressortit à un exil à travers lequel le fils rencontre l'Un hors père.

Pourtant, même Abraham, dont le renoncement au sacrifice du fils est commémoré tous les ans par les Musulmans, n'est pas exempt des errements attachés à la figure du père. Car le texte coranique présente une version de la tentation d'Abraham de sacrifier l'un de ses fils (sans déterminer si c'est Issac ou Ismaël) qui diffère de celle du judaïsme et du christianisme, et s'inscrit dans l'optique qui vient d'être retracée, tout en faisant de l'erreur du père, un ressort spirituel majeur.

En effet, dans la version coranique de cet épisode, Abraham ne décide pas d'emmener son fils au sacrifice par un acte prémédité ; ce n'est pas non plus Dieu qui lui suggère ou lui ordonne de l'immoler. Le désir sacrificiel du père est localisé dans le rêve, et c'est sous l'effet de son rêve qu'Abraham s'adresse à son fils en ces termes: "Mon cher fils, je me vois en songe, en train de t'immoler...» Le texte met alors en scène un fils se soumettant au désir de son père : «Mon cher père », répondit-il, «fais ce qui t'est ordonné...»; puis, un père s'apprêtant à commettre l'infanticide: "Après que tous deux se furent soumis 
et qu'il eut placé l'enfant front contre terre...»; quand Dieu interrompt in extremis l'acte : "Nous lui criâmes: "ô Abraham! tu as cru en ton rêve!" "18

Dans le cri de Dieu arrêtant l'infanticide, s'entend une réprobation qui vise le fait qu'Abraham a adhéré aux images de son rêve. S'appuyant sur cette interpellation, Ibn Arabî, l'une des plus grandes figures spirituelles de l'islam (XIII'), a proposé une théorie très élaborée de l'interprétation du rêve et du sacrifice, qui nous donne la possibilité de penser d'une manière décisive les ressorts spirituels de la question du père dans la version islamique du monothéisme. Cet auteur relève en effet, que la réprobation de Dieu ne peut avoir d'autre signification que le défaut d'interprétation de son rêve par Abraham. C'est parce que le père commet l'erreur de ne pas interpréter son rêve, qu'il en vient à vouloir tuer son fils, et que Dieu intercède pour lui substituer le bélier ${ }^{19}$. Le sacrifice aurait donc la fonction de pallier à une interprétation manquante du père.

Mais quelle est la cause du défaut d'interprétation du père ? Ibn Arabî avance l'explication suivante: "L'enfant est l'essence de son générateur. Lorsque Abraham vit dans un songe qu'il immolait son fils, il s'agissait en fait de se sacrifier lui-même ${ }^{20}$. Ainsi, si l'on considère que l'essence ('Ayn) contient toutes les possibilités d'être, comme le précise Ibn Arabî, on comprend qu'Abraham a éludé l'interprétation de son rêve pour se soustraire à la limitation de sa toute puissance. Le rêve du père de tuer le fils, recèle le désir de sacrifier l'essence génératrice avec laquelle il restait confondu. Or, sacrifier l'essence, c'est accepter «l'odeur de l'existence » (Râ'ihatou al-Wujûd), ou bien encore, consentir à la détermination de l'essence en un lieu (Hulûl), selon les termes d'Ibn Arabî. Cesser d'occuper tous les lieux possibles, tel serait le devenir père. Il n'y a de père qu'en tant qu'existant (Mawjûd), puisque le générateur relève de l'essence qui est l'être, l'Un, Dieu lui-même. On peut tirer ici une triple conséquence : d'une part, le concept d'essence équivaut à celui de jouissance, au sens de la jouissance absolue. D'autre part, l'interprétation aurait la fonction de faire advenir à l'existence, ce que le rêve dans son contenu manifeste dérobe, à savoir que le désir est le désir de sortir de l'essence. Enfin, ce qu'on appelle «père » est un existant qui se différencie du générateur, du créateur ( $k h a ̂ l q$ ) ou de l'être.

Dans cette optique, le père est un procréateur qui ne peut coïncider avec le Dieu créateur, si ce n'est dans le fantasme de toute puissance du père. Ibn Arabî écrit : "Le songe relève de la présence imaginative qu'Abraham n'a pas interprétée. C'était en fait un bélier qui apparut en songe sous la forme du fils d'Abraham. Aussi Dieu racheta-t-il l'enfant du fantasme (Wahm) d'Abraham, par la grande immolation du bélier, ce qui était l'interprétation divine du songe, dont Abraham n'a pas été conscient (Lâ Yach'ur).» ${ }^{21}$

18. Coran, XXXVII,101-112.

19. Ibn Arabî, La sagesse des prophètes, trad. T. Burckhardt, Paris, Albin Michel, 1974, pp. 67-73.

20. Op. cit., p. 67.

21. Ibn Arabi, op. cit., pp. 87-88. 
En somme, le fantasme d'infanticide chez le père, dissimule le désir de tuer le père de la toute puissance, mais comme le père en manque l'interprétation, c'est Dieu qui la rétablit par la substitution du bélier. On constate ici qu'Ibn Arabî propose une théorie très proche de l'interprétation freudienne de l'animal du sacrifice, que J. Lacan avait reprise en soulignant que le bélier est une figure du père de la jouissance absolue ${ }^{22}$.

Le procès de la défaillance du père serait donc le suivant : le père désire être l'essence, en tant qu'il reste confondu avec le fils, mais lorsqu'il cherche la séparation, il en méconnaît la valeur symbolique et veut se la donner en s'infligeant le meurtre réel du fils. À suivre Ibn Arabî, l'islam pense le père à partir de Dieu et non l'inverse. Ce dernier apparaît comme un Dieu qui rattrape la méprise imaginaire du père sur l'altérité, à travers le rétablissement d'une herméneutique du rêve qui fait surgir le symbole manquant dans la réalité. C'est en ce sens, qu'il est créateur et protecteur du fils, à l'encontre de la toute puissance de son père procréateur. Car pour Ibn Arabî, le père procréateur est, par sa nature primordiale, un animal; ou plus exactement, du point de vue de la logique de la procréation, le père de l'homme est l'animal. C'est pourquoi, à défaut d'interprétation, le sacrifice permet la séparation de cette origine, c'està-dire l'advenu à l'odeur de l'existence par l'immolation de l'animal. Ce cheminement aura permis de répondre à la question que pose Ibn Arabî au tout début de son texte: "Comment donc le bêlement du bélier et la parole humaine se valent-ils ?» ${ }^{23}$ Nous pouvons maintenant faire ce raccourci : c'est dans la mort que la voix du bélier devient parole.

$$
- \text { III - }
$$

Cette recherche sur le père a été impulsée par l'une des rares réflexions de Freud à propos de l'islam, qui vient sous sa plume dans Moïse et la religion monothéiste, ainsi que nous l'évoquions tout au début. Il écrit : «La récupération (Wiedergewinnung) de l'unique et grand Père-originaire (Urvater) produisit chez les Arabes une extraordinaire élévation de la conscience de soi, qui conduisit à de grands succès temporels mais s'épuisa aussi en eux. Allah se montra beaucoup plus reconnaissant à l'égard de son peuple élu que jadis Yahvé à l'égard du sien. Mais le développement interne de la nouvelle religion s'immobilisa bientôt, peut-être parce qu'il manquait l'approfondissement qui produisit, dans le cas juif, le meurtre perpétré sur le fondateur de la religion.»

Je ne reviendrai pas ici sur les détails de la discussion que j'ai entamée avec cette proposition ${ }^{24}$, si ce n'est pour souligner que les précédents développements semblent contredire l'hypothèse sur "La récupération (Wiedergewinnung) de l'unique et grand Père-originaire (Urvater)...». La séparation entre un Dieu

22. J. Lacan, Séminaire XI, inédit, séance du 20 novembre 1963.

23. Op. cit., p. 85.

24. Fethi Benslama, op. cit., pp. 115-120. 
créateur et un père procréateur, entre ce dernier, en butte à la figure du père de la jouissance absolue, et le premier en posture d'un Dieu herméneute, garant de la fonction symbolique défaillante du père, indique plutôt que l'islam pense la spiritualité à partir d'une divinité qui est en retrait du père imaginaire de l'origine.

Si l'islam est bien une religion du fils, c'est pour autant que le fils est sauvé de son propre père qui ne parvient pas à se séparer d'un père animal dont le rêve est porteur. Certes, le rêve contient aussi le désir de s'affranchir du désir animal. De ce point de vue, il condense deux désirs : l'un du côté de l'animal, l'autre du côté d'une libération terrible, puisqu'elle consiste à tailler dans la chair du fils. Le Dieu de l'islam apparaît bien comme une critique du père, comme interprétation de son désir, afin de relever le fils. Il consent au meurtre de l'animal dans le père; bien plus, il propose le meurtre du père de la jouissance en le symbolisant par le bélier, lequel n'est pas donc le substitut du fils, comme on le dit souvent, mais bien celui du père. Par le sacrifice de l'animal, le père accède à la vérité symbolique de son désir. Il se supprime comme origine. Je pense ici à la phrase de Hegel : «les parents sont pour l'enfant l'origine qui se supprime ${ }^{25}$. Dans cette optique, l'être du symbole n'est ni du côté du père, ni du côté du fils, même s'il sauve ce dernier de la cruauté du premier. Il est capacité de transposition: que le bélier puisse être l'animal et le père primitif, le symbole et la chose sanglante, le rêve (d'Abraham) et son interprétation (divine), entre image, mot et chose.

Ce que j'appelle ici transposition, Ibn Arabî a tenté d'en faire une théorie de la créativité des formes comme propriété intrinsèque de l'être ou de Dieu. Alors que ce dernier est conscient des multiples formes qu'il peut prendre en toute chose, l'homme n'a qu'une conscience restreinte de la trans-formation. "Nous ne pouvons même pas voir notre propre forme spirituelle » ${ }^{26}$, écrit-il. C'est en ce sens, qu'Abraham, pourtant prophète, ne voit pas la forme spirituelle du père et qu'il doit en passer par tant de métaboles. La raison fondamentale pour Ibn Arabî est la suivante : "Car Dieu n'est jamais inconscient (bi lâ Chu'ur) de rien, tandis que le sujet est nécessairement inconscient de telle chose en rapport avec telle autre. ${ }^{27}$ Aussi, le sujet humain est-il toujours surpris de voir la transposition des choses en autres choses, y compris de lui-même.

Telle est brièvement exposée la théorie d'Ibn Arabî du père, qui le sépare radicalement de Dieu, lequel est pensé comme une énergie de transposition des formes à laquelle l'homme n'a pas accès pleinement, du fait qu'il a un inconscient. L'inconscient d'Ibn Arabî n'est pas l'inconscient freudien, bien qu'il le frôle souvent, il est la condition du voilement et du dévoilement spirituel des multiples formes de l'homme. p. 24.

25. G.W.F. Hegel, La Phénoménologie de l'esprit, trad. Jean Hyppolite, Aubier, 1941, t. 2,

27. Op. cit., p. 97. 
Ibn Arabî n'a pas inventé cette théorie à partir de rien. Il l'a déduite du texte coranique et du discours du fondateur de l'islam. L'hypothèse que j'ai proposée dans la recherche précédemment mentionnée ${ }^{28}$ est que le fondateur de l'islam hérite d'une situation généalogique exposée dans La Genèse où il apparaît que la filiation d'Abraham par Ismaël est le fruit d'une fécondation naturelle d'Agar, alors que pour Isaac, Dieu dût intervenir dans la procréation, puisque Sarah avait plus de soixante-dix ans. C'est cette même opération qui va se répéter avec Marie pour engendrer Jésus. Le père réel pour l'islam est donc Abraham, tandis que le Dieu créateur reste éloigné de la procréation. Dans le judaïsme et le christianisme, Dieu est à la fois créateur et procréateur, tandis qu'Abraham comme Joseph sont des pères symboliques. Ce positionnement généalogique dans la première écriture du père du monothéisme explique à mon sens, que le Dieu de l'islam n'est pas un père. Curieusement, les études comparées du monothéisme n'ont jamais relevé cet aspect essentiel. L'autre fait majeur du récit biblique est qu'Abraham renvoie son fils Ismaël et sa mère dans le désert, les exposant à la mort, n'était l'intervention divine pour les sauver. La figure du père est donc marquée à l'intérieur de l'islam par la question de l'abandon, qui redouble la tentation du sacrifice du fils, quand bien même, le texte coranique met en scène une réconciliation entre Ismaël et son père lors de la reconstruction du temple de la Mecque. Il me semble que cette situation a conduit le fondateur de l'islam à renoncer à l'idéalisation du père, pour dégager d'emblée la conception d'un Dieu qui est être, source d'une fonction symbolique séparée et séparatrice du père et du fils.

Si Dieu rétablit la métaphore paternelle, il échappe à l'ontologie métaphorique du père, non seulement parce qu'il est interdit de l'appeler «père », mais il est impossible de le nommer proprement. En effet, Allah n'est pas un nom comme Yahwé et Jésus. Il s'agit en langue arabe de la contraction de l'article indéfini $A l$ et de Ilâh qui signifie dieu. Allah désigne Ledieu. C'est ce qui a fait écrire à Joseph Chelhod dans Les structures du sacré chez les Arabes : "Si les juifs ont fini par donner à leur dieu suprême un nom qui n'en est pas un (Yahwé, celui qui est), les Arabes ont laissé le leur pratiquement sans nom. ${ }^{29} \mathrm{La}$ théologie négative qui s'est constituée à l'intérieur de l'islam, à partir du $\mathrm{IX}^{\mathrm{e}}$ siècle, se fonde sur cette impossibilité de nommer Dieu, et va jusqu'à considérer le texte coranique comme un tissu métaphorique qui n'est pas l'œuvre d'une révélation de Dieu, ainsi que le dogme de l'islam le stipule, mais d'une inspiration divine écrite par des mains humaines.

Fethi BENSLAMA

Université Paris 7 - 107 rue Faubourg St-Denis BP 120 - 75463 Paris Cedex 10

28. Op. cit., pp. 139-147.

29. Joseph Chelhod, Les structures du sacré chez les Arabes, Maisonneuve \& Larose, 1964, p. 7 . 
Fethi Benslama - D'un renoncement au père

Résumé : L'islam a-t-il tenté de produire dans son édifice spirituel un renoncement au père pour constituer la foi en Dieu ? Cette étude propose une lecture de la construction symbolique islamique à partir de cette question. Partant de l'impératif qui sépare Dieu de toute métaphore paternelle et de toute idée de procréation, notre recherche a essayé d'interroger le texte coranique dans son rapport avec le texte biblique, pour déterminer les raisons d'une divergence de fond entre l'islam et les deux autres monothéismes. Nous avons montré que le problème trouve sa racine dans La Genèse où le fondateur de l'islam a levé l'articulation généalogique à Abraham pour inscrire sa fondation à partir du grand récit de la paternité et de l'alliance. Mais ce récit l'a contraint à une interprétation qui distingue la métaphore paternelle de Dieu. Les conséquences de cette conjecture sont nombreuses, tant du point de vue éthique, qu'au niveau de la théorie du monothéisme.

Mots-clés : Islam - Père - Dieu - Création - Procréation - Métaphore - Impossible Renoncement - Monothéisme.

\section{Fethi Benslama - Giving up the Father}

Summary : Has islam tried to meet its spiritual structure with a ressouncement of the father tou constitue faith in God? From that question, this study porposes a way for reading the symbolic islamic construction. From the requirement that God is separated from any paternal metaphore and from any idea of procreation, we have tried in our reserch to put in perspective the Koranic text and biblical text, to determine the reasons of an in-depth discrepency betxen islam and the two other monotheisms. We have shown that the problem find its root in the book of Genesis where the founder of Islam has gone up to Abraham to set his foundation from the big account of parternity and of alliance. But this account has forced hin to an interpretation which distinguishes the paternal metaphore from god. The consequenses of this conjecture are numerous at the level of ethic as well as that of the theory of monotheism.

Key-words : Islam - Father - God - Procreation - Metaphore - Impossible - Renouncement - Monotheism. 